
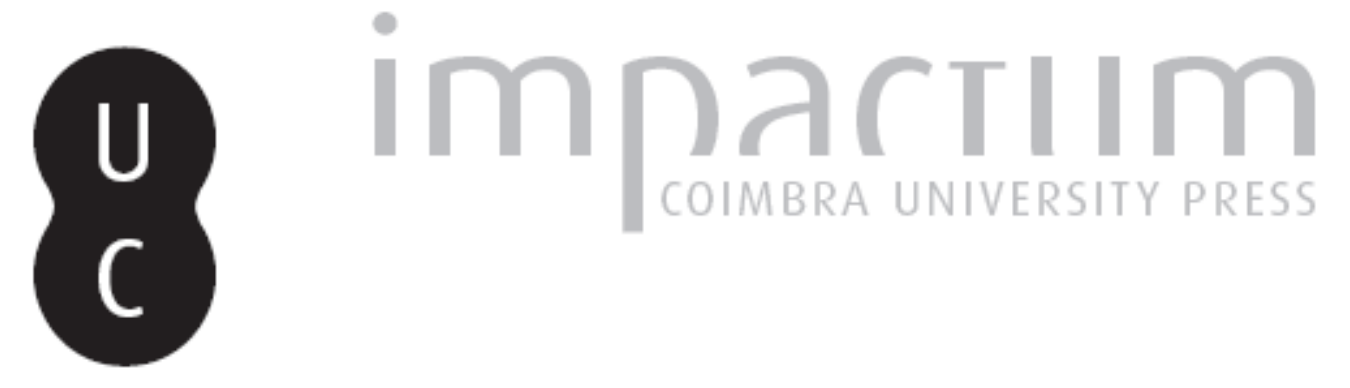

\title{
Los formularios teonímicos, Bandus con su correspondiente femenino Bandua y unas isoglosas célticas
}

Autor(es): $\quad$ Stempel, Patrizia de Bernardo

Publicado por: Faculdade de Letras da Universidade de Coimbra

URL persistente:

URI:http://hdl.handle.net/10316.2/37689

DOI:

DOI:http://dx.doi.org/10.14195/1647-8657_42_7

Accessed : $\quad$ 26-Apr-2023 13:41:39

A navegação consulta e descarregamento dos títulos inseridos nas Bibliotecas Digitais UC Digitalis, UC Pombalina e UC Impactum, pressupõem a aceitação plena e sem reservas dos Termos e Condições de Uso destas Bibliotecas Digitais, disponíveis em https://digitalis.uc.pt/pt-pt/termos.

Conforme exposto nos referidos Termos e Condições de Uso, o descarregamento de títulos de acesso restrito requer uma licença válida de autorização devendo o utilizador aceder ao(s) documento(s) a partir de um endereço de IP da instituição detentora da supramencionada licença.

Ao utilizador é apenas permitido o descarregamento para uso pessoal, pelo que o emprego do(s) título(s) descarregado(s) para outro fim, designadamente comercial, carece de autorização do respetivo autor ou editor da obra.

Na medida em que todas as obras da UC Digitalis se encontram protegidas pelo Código do Direito de Autor e Direitos Conexos e demais legislação aplicável, toda a cópia, parcial ou total, deste documento, nos casos em que é legalmente admitida, deverá conter ou fazer-se acompanhar por este aviso.

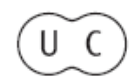


CONIMBRIGA

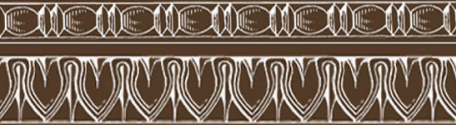

NSTITUTO DE ARQUEOLOGI

I

VOLUME XLII - 2003

A C U L D

UNIVERSIDADE DE COIMBR 
Patrizia de Bernardo Stempel

Universidad del País Vasco/Euskal Herriko Unibertsitatea

LOS FORMULARIOS TEONÍMICOS, BANDUS CON SU CORRESPONDIENTE FEMENINO BANDUA Y UNAS ISOGLOSAS CÉLTICAS

“Conimbriga" XLII (2003) p. 197-212

RÉSUMÉ: A travers de la typologie des formulaires théonymiques se démontre qu'aussi dans le cas de la divinité en question il y a trois types syntactiques du déterminant: 1. adjectival; 2. au génitif du pluriel; 3 . au génitif du singulier. Les adjectifs aussi que les génitifs du pluriel - souvent sans notation de la nasale finale dans la zone en question - peuvent avoir des formes épichoriques (-acui, -aikuil-egui; -ko(n)/-go(n), -aeko(n)/-aego(n)) ou latines $(-e(n) s i ;-u(m))$.

En plus il est démontré que les dédicaces peuvent avoir la forme d'un datif du singulier d'un thème en $-u$ - masculin et flexion celtibérique $(-(u) e i)$ ou latine classique $(-(u) i)$ ou périphérique $(-u)$, mais aussi la forme d'un datif du singulier d'un thème en $-\bar{a}$ et flexion lusitanienne et italique $(-a)$ ou latine classique ou vulgaire (-ae > -e), et même d'un datif masculin redéterminé par la désinence féminine du latin $(-(u) i+-a e)$. La chute de $-w$ - après nexus consonantique $(n d w V>n d V)$ aussi que les théonymes avec une variante masculine et une féminine étant fréquent en celtique, il est proposé que Bandus, avec sa forme de thème en $-u$ - reservée en indo-européen à des concepts sacrés et proche du v.-ind. bandhuh 'parenté; relation', aie été une divinité originairement celtique avec motion Bandua aux féminins en $-\bar{a}$.

RESUMEN: A través de la tipología de los formularios teonímicos se enseña que también en el caso de la divinidad en cuestión hay tres tipos sintácticos del determinante: 1 . adjetival; 2 . en genitivo de plural; 3 . en genitivo de singular. Tanto los adjetivos como los genitivos de plural - a menudo sin notación de la nasal final en el área en cuestión - pueden 
tener formas epicóricas (-acui, -aikuil-egui; -ko(n)/-go(n), -aeko(n)/ l-aego(n)) o latinas $(-e(n) s i ;-u(m))$.

Se enseña además que las dedicaciones pueden tener la forma de un dativo singular de un tema en $-u$ - masculino y flexión celtibérica $(-(u) e i)$ o latina clásica $(-(u) i)$ o periférica $(-u)$, pero también la forma de un dativo singular de un tema en $-\bar{a}$ y flexión lusitana e itálica $(-a)$ o latina clásica o vulgar $(-a e>-e)$, e incluso la de un dativo masculino redeterminado con la desinencia femenina del latín $(-(u) i+-a e)$. Siendo tanto la desaparición de - $w$ - detrás de nexo consonántico $(n d w V$ $>n d V$ ) como los teónimos con una variante masculina y otra femenina frecuentes en el celta, se propone que Bandus, con su forma de tema en $-u$ - reservada en indoeuropeo a conceptos sagrados y cercana del ind.ant. bandhuh 'parentesco; relación', haya sido una divinidad en origen céltica con moción Bandua a los femeninos en $-\bar{a}$. 


\section{LOS FORMULARIOS TEONÍMICOS, BANDUS CON SU CORRESPONDIENTE FEMENINO BANDUA Y UNAS ISOGLOSAS CÉLTICAS ${ }^{1}$}

\section{0. ¿Problema?}

El supuesto problema del género ${ }^{2}$ de lo que se ha llegado a denominar "uma divindade indígena como Band-" ("na imposibilidade de poder determinar a forma nominativa": de Hoz/Fernández 200246 y 45) desaparece, y con eso el problema de determinar su forma originaria, si analizamos la escasa treintena de dedicaciones involucradas teniendo en cuenta dos circunstancias: a) que a la hora de 'conectar' una divinidad cualquiera con un epíteto no se suele utilizar siempre la misma estructura sintáctica, sino que se aprovechan distintos tipos de formularios $(\S 1)$; b) que el territorio occidental y en particular el lusitano, en el que se hallan dichas inscripciones (mapa 6 en Marco 1999; mapa 14 en Prósper 2002; Olivares 2000-2001 251, 2001 59, 200258 y 86134 159; de Hoz/Fernández 2002 47) se caracteriza por la aparición de unos rasgos morfofonológicos peculiares ( $\$ 5)$. Si a estos aspectos añadimos que la desaparición de la semivocal $* w$ detrás de nexos consonánticos (como la que se aprecia en unas cuantas variantes del tipo teonímico en cuestión halladas - según Pedrero 1997/1999 537s. al sur del Duero) es una isoglosa descrita para las lenguas célticas (§ 4), es posible plantear un marco interpretativo general para los datos hasta

1 Trabajo realizado en el marco del proyecto F.E.R.C.AN de la Österreichische Akademie der Wissenschaften. Deseo agradecer a los amigos y colegas J.A. Arenas-Esteban y F. Marco su preciosa ayuda con el texto español.

2 Hablo aquí simplemente desde el punto de vista lingüístico, o sea me refiero específicamente al Genus, coincidiendo con J. d'Encarnação en el considerar que sería bizantino querer atribuir a la(s) divinidad(es) un Sexus "que, na verdade, o não possuem" (2002 525). 
ahora disponibles y, en nuestra opinión, injustamente considerados insolubles por los paleohispanistas. En cuanto al corpus de las inscripciones conocidas - que este artículo no pretende de ninguna forma modificar - se remite a los recientes trabajos de Pedrero (1997/1999 y 1999/2001), Prósper (2002) y de Hoz/Fernández (2002)³.

\section{Los formularios teonímicos. ${ }^{4}$}

El determinante por el que se especifica la forma cultual de la divinidad venerada puede corresponder básicamente a dos tipos semánticos, cuya repartición con respecto a las MATRES en la Narbonensis fue identificada por Lejeune (1982 107). Se trata de los que él llama

$\alpha$ ) épiclèses descriptives: indicación de una facultad o rasgo del carácter divino en cuestión, como en el caso de las Matres Dervonnae de las encinas o de Juppiter Solutorius y Repulsorius (tratados últimamente por Zaccaria y Beltrán 2000/2002);

乃) épiclèses toponymiques: indicación de pertenencia a un grupo de población o ciudad.

Nótese que con respecto a la Hispania antigua: "Die bisher erschienenen Arbeiten stellen Beziehungen vor allem zu Ortsnamen her (z.B. Naviae Sesmacae, Deo Durbedico, Laribus Tarmuncebaecis Ceceaecis); im allgemeinen all jene, die die Elemente -aico und -briga aufweisen [...] Es liegen auch Beispiele vor, die klar mit Ethnonymen in Beziehung stehen, was uns zu Schutzgottheiten mehr oder minder umfangreicher menschlicher Gemeinschaften führt (Numina Lapitearum [...]), oder mit Personennamen wie die [...] Lares Cereneaeci und Cumelani, die sich demnach auf Hausgötter beziehen" (González Rodríguez 2000/2002 51).

En las lenguas indoeuropeas, y específicamente en las inscripciones latinas y célticas, las epíclesis descriptivas se suelen expresar sintácticamente por medio de un atributo o aposición ( $1^{\text {er }}$ tipo sintáctico), es decir, de un adjetivo o sustantivo que concuerda con el teónimo en

3 Los últimos tres faltan, por razones cronológicas, en Olivares Pedreño 2002, que por su parte también recoge toda la información sobre lo que él llama "Bandua, el protector de la comunidad" (Cap. 12).

4 Los teónimos citados sin bibliografía particular están recogidos en el RDG. 
género, número y caso (p.ej. MARS CIC-OLLOS 'M. todo-muslo' en los dativos Marti Cicollui). Sin embargo, a la hora de indicar la pertenencia étnico-geográfica de la divinidad (epíclesis del tipo ß), se hallan tres diferentes construcciones: además del $1^{\text {er }}$ tipo sintáctico, que en el caso en cuestión aprovecha un adjetivo derivado desde un topónimo o desde un etnónimo (así en Marti Cemenelo, Mercurio Arverno), hay también una construcción donde el teónimo es acompañado por el sustantivo que indica las personas que le veneran en genitivo de plural $\left(2{ }^{\circ}\right.$ tipo sintáctico), como en los casos de las Senonum Matres o del Genius Nerviorum y, respectivamente, en el del Genius Tongobrigensium y de la dedicación Nimidi Fiduenearum (González Rodríguez 2000/2002 51); por último, otra construcción aprovechada para este segundo tipo de epíclesis es aquella donde el teónimo va acompañado por un nombre de lugar - a menudo de una ciudad - en genitivo de singular ( $3^{\text {er }}$ tipo sintáctico), como en la dedicación Munidi Eberobrigae Toudopalandaigae (González Rodríguez 2000/2002 50) o posiblemente en deae Mogontiae.

Es importante subrayar que el determinante de un teónimo específico no estaba necesariamente asociado a un único tipo sintáctico, como se puede apreciar p.ej. en el caso de la diosa céltica de la noche venerada en Hispania, ATAECINA (la etimología puesta al día en NWÄI 80), en cuyas menciones (Abascal 1995) se aprecian al mismo tiempo los tipos sintácticos

$1 \alpha$ : Adaecinae Sanctae

1ß: Turibri $(g) e(n s i)$ Adegin(a)e

3: Ataecina Turobrigae

Otro ejemplo de dicha variación se observa en las

Matronis Gesahenis (tipo sintáctico $1 \beta$ ) vs. las

Matronis Gesationum (tipo sintáctico 2).

Incluso cuando la misma divinidad va acompañada por más de una epíclesis, tampoco es necesario que se utilice el mismo tipo sintáctico, cf. la dedicación deo Marti Medocio Campe(n)sium.

\section{La diosa Bandua. ${ }^{5}$}

Del mismo modo que los formularios no tienen por qué ser, y de hecho no son monolíticos, las formas lingüísticas en las que se mani-

5 Los números en corchetes detrás de las formas citadas indican su ubicación con respecto a los trabajos de de Hoz/Fernández 2002 (HF), Pedrero 1999/2001 (Pe) y Prósper 2002 (Pr). 
fiesta el teónimo reflejan una gran variedad, dependiendo de factores tanto intra- como extralingüísticos.

Pese a la rotunda negación por algunos autores de que haya existido una diosa BANDUA, la pátera de Badajoz nos muestra una divinidad iconográficamente femenina; y no tenemos por qué excluir que sea una divinidad igualmente femenina la que se venera en las dedicaciones con el morfema latino de dativo singular de los temas en $-\bar{a}$. Este tipo se encuentra en tres variantes: la más arcaica, aunque ya con la conocida monoptongación latina del diptongo, es Bandue ([DF 8; Pr 260] dativo sin epíteto en una inscripción desaparecida "não anterior à segunda metade do século II" de la era imperial, Redentor 2002 51). Más desarrollada es la variante que ha perdido, además del diptongo, también la $w$ postconsonántica, o sea el dativo Bande. A este respecto cabe resaltar que la desaparición de la sonante en un contexto fonético de dicha estructura no es nada singular en el contexto de las lenguas célticas ( $\$ 4)$, como ya he tenido ocasión de subrayar (2002 91 n.11). Un subtipo de esta variante es además el dativo Bane (sin epíteto, desde el Distr. de Castelo Branco [Hisp. Epigráfica 4 1994378 n. ${ }^{\circ}$ 1034] y Zamora [Pr 268]), donde se aprecia la asimilación del grupo $n d$ a $n(n)$ descrita para el celtibérico y otras áreas del celta hispano (De Bernardo Stempel 2002103 y 117). En la tercera modalidad, el ditpongo -ae del dativo latino clásico está preservado, pero se junta - en el único y además desaparecido hallazgo Bandiae [DF 24; Pr 260s.] - a una semivocal $-i$ - en vez de la $* *$ - $u$ - esperada; parece por lo tanto una contaminación con el dativo Bandi del correspondiente teónimo masculino. En esta dirección podría apuntar también la forma del epíteto de agrupación familiar Apolosego ${ }^{\mathrm{N}}$ ([Pe 546s.] en genitivo de plural) si en el compuesto indígena que tiene como determinatum el adjetivo sego - 'fuerte' la base Apulu - hubiese sido influenciada por el nombre de APOLLO.

Hay además otro tipo de dedicaciones en dativo, donde la divinidad femenina se cita aprovechando el morfema -a\# de tipo itálico de los temas en $-\bar{a}$ (De Bernardo Stempel 2000 55s.), que se halla también en latín dialectal y que last not least nos enseñan los dativos femeninos de teónimos en inscripciones lusitanas (p. ej. de la céltica Iccona en Cabeço das Fráguas): éste es el caso cuando los teónimos dependen sintácticamente de fórmulas del tipo v.s. como en IRG-04, 00089 desde Eiras (cit. aquí según Clauss/Slaby) y en la inscripción de Alenquer ${ }^{6}$,

${ }^{6}$ Leída como I. Eranigi Ommia Bandua Horrico v.als por M. Alves-Dias en el 4. Workshop F.E.R.C.AN (próximamente en Spickermann/Wiegels). 
donde Bandua parece además encontrarse junto con otros dativos. A veces sin embargo, como en el ejemplo de la Bandua de San Amaro [DF 2], el contexto no permite excluir que se trate de un nominativo, aunque también aquí el dativo en -a\# parece más probable, siendo el nombre de la diosa de Lanobriga seguido únicamente por el nombre del dedicante al nominativo.

Puede, por fin, que haya además una forma de genitivo singular Banduae en la epíclesis de otra divinidad, el DEUS VEXILLOR() MARTIS, al que se le identifica también como SOCIUS BANDUAE; "En cualquier caso, la autenticidad" "dieser derzeit verschollenen Quelle" "es materia de discusión” (resp. Prósper 2002259 n.6 y Marco 1998 55), así que de Hoz/Fernández 2002 la escluyen de su listado, mientras que en Olivares Pedreño 200288 se encuentran "argumentos que apoyan la tésis de que esta inscripción no es falsa".

Si clasificamos ahora los hallazgos con divinidad femenina y epíteto en función de los formularios teonímicos empleados, podemos distinguir los tipos sintácticos:

$1 \beta)$, con adjetivo atributivo de pertenencia:

Band(ua) Araugel(ensis) [DF 49]

datBand() Ocel(ensi) [DF 13]

La primera inscripción quedaría así integrarada por hallarse sobre una pátera con figura femenina, aunque pudiera tratarse también de un dativo; el epíteto es confirmado por los castellanis Araocelensibus de otra inscripción (de Hoz/Fernández 2002 46s.).

La integración del segundo epíteto (cf. Hisp. Epigráfica 31993 192 n. ${ }^{\circ}$ 494; nótese que hay también la lectura Bandi Oge $[\operatorname{Pr} 110$ y 260], cf. Hisp. Epigráfica 41994399 n. ${ }^{\circ}$ 1100) es puramente analógica: el adjetivo detoponímico se atestigua de hecho tanto en la forma latinizada Ocelenses (además epigráficamente: Vicani Ocel[e]n/n[se]s, Prósper 109s.) como en la forma indígena *Okel-ākyo- (en el antropónimo genOcelaici y en el epíteto del LAR OCAELAEGO), aunque dedicante y ejecutor de la dedicación pertenezcan en este caso indudablemente al entorno céltico (Camalos Ulpinif. y su hijo Celtius).

2), con genitivo plural de un nombre de agrupación:

dat Bandue + gen.pl Aetobrico ${ }^{N}$ [DF 4; Pe 544; Pr 257s.]

Bandue $\quad B{ }^{\text {'olecco }}$ [DF 1; Pr 259]

Bandue $\quad$ Cadogo $^{N}$ [DF 7; Pe 549; Pr 258]

Bandue Roudeaeco ${ }^{N}$ [DF 26; Pe 551s.; Pr 266]

Bandue Veigebreaego ${ }^{N}[\mathrm{DF} 3$; Pe 553; Pr 259] 
Tras lo dicho hasta aquí y en el $\S 5$, se puede suponer que las epíclesis de este grupo no representen dativos temáticos singulares de atributos masculinos sino más bien genitivos de plural en $-o(m)$ o eventualmente en $-o(n)$. Pensamos por lo tanto estar en presencia de grupos de población como los *Veikyobrigā kyōs (*Veikyobrigākyoi en la fase lingüística más moderna), procedentes de la *Ciudad combativa (*Veikyobriga), y los *Roudyākyōs/oi, que aparecen como vicani Roud() en una inscripción de Casar de Cáceres (Olivares Pedreño 2002 34) y evocan a los *Valientes o Rojos. En el caso de los ciudadanos *Aetobriges (para la grafía con velar sorda cf. los ejj. recogidos por mí 2002119

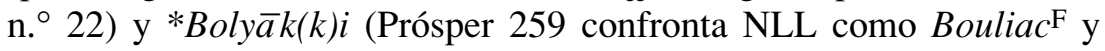
Boulich ${ }^{\mathrm{D}}$; la lectura $C^{?}$ olecco en de Hoz/Fernández se relacionaría más bien con los Coelerni y su ciudad Coeliobriga, cf. Albertos 1990 134), como también en el caso de los *Kadoki ('Honrados, Nobles', a confrontar con los *Kadyoki o *Kadyaki atestiguados en el gen.plCadde$\left(u n^{7}\right.$ ), la falta misma de un sufijo derivativo de adjetivos nos indica que no estamos en presencia de atributos sino directamente de los sustantivos que designan grupos étnicos o simplemente sociales. dat Bande + gen.pl $R$ ? aeico ${ }^{N}[\mathrm{DF} 9]$

Bande + genn.pllVelugo ${ }^{N}$ Toiraeco $^{N}$ [DF 15; Pe 554; Pr 264]

La afirmación de ciertos autores de que las dos epíclesis de Bande Velugo $^{N}$ Toiraeco $^{N}$ no puedan ser igualmente étnicas, es una simple petitio principii, porque conocemos fórmulas onomásticas antiguas $\mathrm{y}$ modernas donde un primer apellido necesita ser determinado por otro que especifica de cuál rama de una numerosa familia se trata (Ortiz de Urbina vs. Ortiz de Zárate, Ferrari da Grado), una costumbre muy difundida en las familias nobles (von Sachsen-Weimar vs. von SachsenCoburg-Gotha, von Habsburg Lothringen etc.); así en el tercer bronce de Botorrita Koitina de los Verzaizokum Kalmikum (col. II, 11. 26s.), Turaios de los Litanokum Kurmilokum ( $¿$ 'de los Galos productores de cerveza?’, col. III 11. 57s.), y quizá el SeKontios de una rama de los Turmogi (TurumoKum Ultatun, col. II 1. 48). La pieza en cuestión puede por tanto estar perfectamente dedicada a la BANDUA a la cual venera la rama de los Toryaki (¿o quizá * Doryaki? cf. la isoglosa n. ${ }^{\circ} 22$ en De Bernardo Stempel 2002 119s., NPP como Dorio, Dorionis y el hecho de que esta-

7 La reconstrucción ofrecida anteriormente por la autora (2002 112) se puede mejorar a la vista de la isoglosa $* y o / y a>e$ en posición átona en áreas del celta italiano y en goidélico (Roma 2002a, en prensa). 
mos en las inmediatas cercanías del Doríou potamoû, Ptol. II.5.2) procedente de los *Veluki (Pedrero 1999/2001 534 confronta la ciudad de O úeloúka en el territorio de los Arevaci).

3), con genitivo singular de nombre de ciudad:

datBandua + gen.sgLanobrigae [DF 2; Pe 544s.; Pr 258s.]

Sobre la ciudad de Lanobriga v. Albertos 1990 p. 138.

\section{El dios Bandus.}

Al discriminar las inscripciones votivas donde aparece la diosa $B A N D U A$, se constata que dentro del conjunto de dedicaciones con teónimos que comparten la misma base derivacional sólo quedan ejemplares que nombran a una divinidad BANDUS, que cabe suponer lingüísticamente masculina.

Hasta el momento la divinidad ha aparecido en dativo, y mayoritariamente en la forma latina de dativo singular de tema en - $u$-. Paralelamente a lo que habíamos constatado para el femenino, también del tipo masculino alla latina se conocen por lo menos dos variantes: la regular Bandui y aquella con simplificación fonética de la $w$ posconsonántica (explicada en el $\S 4$ ), es decir Bandi ${ }^{8}$.

El segundo tipo de dativo que se atestigua corresponde igualmente - como en el caso del teónimo femenino - a una variante conocida dentro del latín mismo, incluso el utilizado por César: se trata del dativo en - $u \#$ de los temas en - $u$ - (Ernout MorphHistLat 67): Bandu Virubri[] (DF 6; el epíteto es leído por otros Virubric y hasta Virubrico, cf. resp. Clauss/Slaby y Albertos 1990 143; Pe 545s.; Pr 259); su escasa frecuencia es un rasgo común a los dos corpora. Desde luego, nada se puede decir de las formas Bandu[] que preceden a Etobrico [DF 5; Pr 258] y a Vordeaeco [DF 11; Pr 260].

El tercer y último tipo de dativo de tema en $-u$ - que nos muestra el teónimo masculino es morfológicamente más arcaico, por tener el mismo morfema de dativo *-uei que se halla en el celta hispano (cf. el Luguei de la inscripción grande de Peñalba ${ }^{9}$ ), aunque con la conocida

${ }^{8}$ Gratuito es el postulado de aquellos autores que hacen de Band $\underline{\boldsymbol{i}}$ y Band $\underline{\boldsymbol{e}}$ variantes fonéticas de una misma forma del teónimo.

9 De Bernardo Stempel 1998 (Clermont, en prensa: § I.9.1); unas nuevas propuestas en 2000a 189. 
supresión de $w$ detrás de dos consonantes: es el *Banduei Brialeacui $>$ Bandei Brialeacui en dos inscripciones desde Covilha. De este tipo puede qu exista también la variante fonéticamente todavía más desarrollada, o sea con $n d>n n$, si la lectura [Ban]nei Picio en Belver $\left(\mathrm{n} .{ }^{\circ} 1\right.$ del cuadro 2 en de Hoz/Fernández 2002; Pr 266; Olivares Pedreño 2002 61) es correcta.

Paralelamente a la clasificación del teónimo femenino con sus epítetos (en el § 2), podemos distinguir los hallazgos del correspondiente masculino BANDUS con epítetos en los siguientes tipos sintácticos:

$1 \alpha / \beta)$, con adjetivo atributivo de calidad/pertenencia:

datBandei + dat.sg Brialeacui [DF 17; Pe 548s.; Pr 261s.],

donde los dos dativos están formulados conforme al habla epicórica. datBandui + dat.sgIt.uicie(n)si [DF 25 y p. 47],

donde los dos dativos están formulados en latín.

dat Bandi + dat.sg Isibraiegui [DF 21; Pe 550; Pr 262]

Bandi + Tatibeaicui [DF 14; Pe 553; Pr 263],

con una mezcla de rasgos latinos y epicóricos. El primero de los dos epítetos parece estar contenido también en las dedicaciones Bandi $I s(i) b[$ [DF 22; Pr 262] y Bandu? i Isi? [DF 23; Pr 262], aunque no podemos saber en cuál de los tipos sintácticos.

2), con genitivo de plural de un nombre de agrupación:

datBandi + gen.pl $[L]$ ongobricu(m) [DF 12; Pe 543s.; Pr 263], donde se dedica mediante fórmula latina al dios BANDUS de los * Longobriges (para la ciudad cf. Albertos 1990 138; la grafía con velar sorda es como se ha dicho arriba hipercorrecta).

datBandi + gen.pl Malunrico ${ }^{\mathrm{N}}$ [DF 28; Pe 550s.; Pr 263]

Bandi Saisabro ${ }^{\mathrm{N}}$ [DF 27; Pe 552; Pr 264],

que nos muestran genitivos epicóricos, según la lectio difficilior del primero, de un grupo de *Malunriges (así ya Blázquez pace Prósper 2002 263 n.8), otra vez con grafía hipercorrecta, y de un grupo de *Sasyabri, cuya estructura se parece a la de los Artabri y Cantabri ${ }^{10}$.

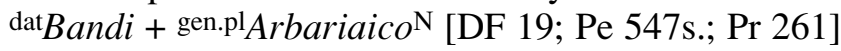

Bandi Oilienaico[m?] [DF 16; Pe 551; Pr 263s.]

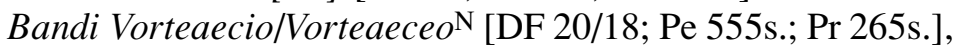
donde, pese a la presencia de sufijos adjectivales y a la concordancia en el género, es también posible ver genitivos epicóricos de gru-

10 Analizada en 2002 107s., donde se ofrece además la traducción "(habitantes) de la/s ciudades de piedra" y respectivamente "cuadradas"). 
pos como los *Arbaryāk-yoi (¿descendientes de los agricultores?), *Olyonākyoi y *Vortyākyoi.

\section{La caída de $* w$ detrás de dos consonantes.}

Como hemos adelantado (2002 91 n.11, aunque con una restitución del teónimo ahora superada), se trata de una isoglosa perfectamente regular en varias áreas y etapas del celta. Así p.ej. en irlandés (NWÄI 218ss.), como resultante de la contraposición entre airl. ${ }^{3}$ mell 'agradable' y ${ }^{1}$ delb 'forma' o selb 'propiedad', o entre ${ }^{*} H R d^{h}$ wo - y *mrwo - que acaban regularmente en ard 'alto' vs. marb 'muerto'. La misma caída se aprecia regularmente en galés, donde ardd se corresponde, con el mismo sentido, al irlandés ard. Lo mismo puede pasar en otros tipos de sílaba pesada, como *swa $d w o$ - que acaba siendo simplemente hawdd 'sencillo, agradable' en galés, frente al más arcaico galo Suaduilla (NP). El galo de la Cisalpina preserva la forma más arcaica en el acusativo de plural artuaš 'piedras' en Todi frente al airl. ${ }^{2}$ art 'piedra', pero dentro del celta continental se hallan al mismo tiempo formas con la semivocal todavía preservada frente a otras con desaparición de la misma; cf. p.ej. las dos variantes de $*$ deksswo-/a 'derecho/a' atestiguadas en el celta hispano: Dessua-eona (cib.) vs. Desso-briga con el desarrollo que será luego del airl. dess gl. dexter.

\section{Los genitivos de plural del celta hispano.}

Las inscripciones en lenguas indoeuropeas procedentes de la Península Ibérica muestran varios tipos de genitivo de plural. El corpus específicamente celtibérico comprende unas cuantas formas arcaicas de genitivo de plural temático en - $\bar{o} m$ (cf. Segaizakōm, Kont(r)ebakōm Karbikōm, Ekualakōm) ${ }^{11}$, una mayoría de formas 'clásicas' en -um, es decir con regular desarrollo fonetico $\bar{o}() \#>u() \#$ (entre otras Abulokum, Balaisokum), y unas raras variantes diatópicas en -un, como en el nombre de los ciudadanos de Olca atestiguado en la leyenda monetal en alfa-

11 Cf. el estudio de conjunto de la flexión nominal en celta continental presentado en Clermont (1998, en prensa); otra $* \bar{o}$ todavía preservada en celtibérico arcaico es la del imperativo claramente visible en la estela de Retugeno (Arenas et al. 2001). 
beto epicórico A. 60 Olkairun (< célt. *olkaryǒ $n \leftarrow$ ie. *olkaryōm) y en el veniakun 'de los familiares' en la tésera de Custodia (cf. la discusión en De Bernardo Stempel 2002 114). Este tercer tipo se presenta, como es de esperar, más numeroso en el corpus hispanocéltico, donde como indicara González Rodríguez ya en el 1986 además del Caddecun y quizá del Ultatun comentados arriba (\$2), encontramos también Alongun, Balatuskun, Bodeccun y Boddegun, Cantabrequn, Celtigun (2002 1.c. y Sastre Prats 2002 83), todos en territorios noroccidentales. También en el corpus hispanocéltico y en territorios afines, se hallan con bastante frecuencia otros genitivos de plural temáticos en -on, como Ateroecon, Belvicon, Sailciecicon.

Evidentemente, cuando los genitivos están - como a menudo ocurre - abreviados, es muy difícil distinguir entre el segundo y el tercer tipo, así como entre el primero (Letondiquom) y el cuarto: es decir, que no se puede saber con certeza si los varios Eburanco(), Turoco() et sim. se tienen que completar con $-m$ o con $-n$. Lo que sí se puede decir es que sus respectivos morfemas no necesariamente prolongan el desarrollo celtibérico de la desinencia indoeuropea, contribuyendo en consecuencia a configurar otras áreas lingüísticas con una parte de isoglosas célticas alrededor de la propia Celtiberia (2002 113s.).

Sin embargo no es eso lo que nos interesa ahora, sino simplemente resaltar la existencia de genitivos de plural paleohispánicos con vocal -o-, y además escritos sin notación de la nasal final, de la misma forma que se conocen genitivos abreviados en $-u$; es decir, que los dos tipos abreviados que hemos identificado en las dedicaciones a BANDUS y a su correspondiente BANDUA (arriba $\S \S 3$ y 2) se hallan independientemente de nuestra divinidad dentro del corpus hispanocéltico. Merece además la pena subrayar que las respectivas áreas de dispersión de los genitivos característicos del celta hispano y de las dedicaciones a $B A N D$ - son parecidas.

\section{Conclusiones.}

De todo esto cabe concluir que exactamente como en el caso de los dobletes de dioses célticos BORMANOS y BORMANA, BELISAMA y BELISAMAROS, CAMULOS/CAMULORIX y CAM(U)LORIGA, MERTRIOS/MERTRONNOS y ROSMERTA ${ }^{12}$

12 Cf. además los grupos de divinidades femeninas acompañados por una divinidad masculina correspondiente, como en la dedicación Glani et Glanicabus desde 
estamos en presencia de un teónimo formalmente masculino (BANDUS) y de su correspondiente femenino $(B A N D U A)^{13}$; los dos aparecen acompañados por determinantes en construcciones sintácticas de varios tipos: a veces atributivos (Bandei Brialeacui) y a veces genitivales (en singular, como en Bandua Lanobrigae, o en plural, como en Bandi [L]ongo$\operatorname{bricu}(m)$, entre ellos varios en <-o\#> que la mayoría de autores presuponía ser del $1^{\text {er }}$ tipo sintáctico).

Aunque no podemos descartar la posibilidad de que la variante masculina haya sido creada más tarde (o sea desde un originario Bandu$a$ derivado como colectivo/abstracto en $-\bar{a}$ de la base *bandu-), parece sin embargo más probable que la forma gramatical originaria del teónimo fuese un masculino y que la variante en femenino se generase en un estadio posterior (como en el caso de BORMANA) por dos razones: 1) $B a n d u-\bar{a}$ corresponde morfológicamente a la moción femenina de un masculino ${ }^{*}$ Bandus $^{14}$ y 2) para el masculino se atestigua todavía la forma de dativo prerromana.

Por ser un tema en - $u$ - es verosimil que Bandus represente desde el punto de vista etimológico el regular correspondiente céltico - aunque aparentemente con grado cero de la raíz - del tema en - $u$ - masculino védico y sánscrito bandhuh 'Verwandtschaft, Genossenschaft, nahe Verbindung, Beziehung' (Mayrhofer EWAIA II/13 208s.); recordaremos a este propósito que al núcleo de los temas en - $u$ - pertenecen tanto en celta como en las demás lenguas indoeuropeas - sobre todo conceptos básicos ("zentrale Begriffe"), a menudo de sentido sacro (NWÄI 93s.). La semántica resultante explicaría por qué prácticamente todas las epíclesis de nuestra divinidad parecen ser de pertenencia social o étnica (cf. Pedrero 1997/1999 541).

Vemos por lo tanto que una interpretación céltica de la divinidad en cuestión permite explicar a la vez todos los rasgos aparentemente singulares del corpus de inscripciones en las cuales se halla, sean

Saint-Rémy-de-Provence (en Clauss/Slaby). Al mismo contexto puede que pertenezcan también Fontanus/a en el caso que representen al dios céltico Belenos de las fuentes (< ie. * $g^{\mathrm{w}}$ elen-o-s, De Bernardo Stempel 2003).

13 Eso no implica evidentemente que estemos en presencia de dos divinidades "separadas", sino más bien de "a mesma sob duas designações sexualmente diferenciadas", apareciendo la divinidad como masculina o femenina "consoante os dedicantes a querem ver" (Encarnação l.c.).

14 Cf. entre otros el NP femenino Katua $(*$ Katu- $\bar{a})$ hallado cerca de Bergamo ${ }^{I T}$ sobre una fusaiola del s. V a.C. (Morandi 2000 16). 
fonológicos (como la desaparición de la $w$ tras sílaba pesada), morfológicos (como los dativos celtibéricos en - $(u) e i$ ), sintácticos (como los genitivos de plural en función de epíclesis etno-toponímicas),

lexicales (el tema en $u$ con valor sacro encaja en el marco de coincidencias lexicales entre celta e indo-iranio ${ }^{15}$ ),

geográficos (la distribución de las variantes fonéticamente más desarrolladas al sur del Duero [Pedrero 1997/1999 538] está de acuerdo con el mayor arcaismo del celta hispano septentrional) e incluso cultuales (como la coexistencia del mismo concepto divino en dos formas, una masculina y otra femenina).

\section{BIBLIOGRAFÍA}

Clauss/Slaby = Epigraphik-Datenbank Clauss/Slaby (www-db.ku-eichstaett.de). NWÄI = De Bernardo Stempel, P. Nominale Wortbildung des älteren Irischen: Stammbildung und Derivation, Tubinga 1999 (= Buchreihe der ZcP 15).

RDG = Jufer, Nicole/Luginbühl, Thierry Répertoire des dieux gauloises, Paris 2001.

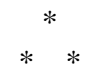

Abascal Palazón, Juan Manuel 1995 «Las inscripciones latinas de Santa Lucía del Trampal (Alcuéscar, Cáceres) y el culto de Ataecina en Hispania» Archivo Español de Arqueología 68 (n. ${ }^{\circ}$ 171-172) 31-105.

Albertos Firmat, Ma Lourdes 1990 «Los topónimos en -briga en Hispania» Veleia 7 131-146.

Arenas, Jesús/De Bernardo Stempel, P./González Rodríguez, Ma C./Gorrochategui, J. 2001 «La estela de Retugenos (K. 12.1) y el imperativo celtibérico» Emerita 69/2 307-318.

Beltrán LlORIS, Francisco 2000/2002 «Iuppiter Repulsor(ius) y Iuppiter Solutorius: dos cultos provinciales de la Lusitania Interior» Veleia 18-19, 117-128.

De Bernardo Stempel, Patrizia 1997 «Celtico e antico indiano: in margine alle più recenti teorie», Bandhu: Scritti in onore di C. Della Casa, edd. R. Arena/M.P. Bologna/M.L. Mayer Modena/A. Passi, Alessandria. Vol. II, 717-734.

EADEM 1998 «Le declinazioni nel celtico continentale: innovazioni comuni al gallico e al goidelico?» Gaulois et Celtique continental (Clermont-Ferrand, 13-16 mai 1998), edd. P.-Y. Lambert/G.-J. Pinault, Paris [en prensa].

15 Un balance sistemático en De Bernardo Stempel 1997. 
EADEM 2000 «Kernitalisch, Latein, Venetisch: ein Etappenmodell», 125 Jahre Indogermanistik in Graz, edd. M. Ofitsch/Ch. Zinko, Graz. 47-70.

EADEM 2000a «Celtib. karvo gortika 'favor amicitiae', rita 'ofrecida', monima 'recuerdo' y los formularios de las inscripciones celtibéricas» Veleia 17 183-189. EADEM 2002 «Centro y áreas laterales: la formación del celtibérico sobre el fondo del celta peninsular hispano» Palaeohispanica 2 89-132.

EADEM 2002a «La ricostruzione del celtico d'Italia sulla base dell'onomastica antica», Onomastica dell'Italia antica (Convegno, Roma 2002), edd. P. Poccetti/St. Verger, Roma [MEFRA, en prensa].

EADEM 2003 «Die sprachliche Analyse keltischer Theonyme» Zeitschrift für celtische Philologie 53, 41-69.

ENCARNAÇÃo, José d' 2002 «O sexo dos deuses romanos» Scripta antiqua, Valladolid. 517-525.

ERNOUT, Alfred 31974 Morphologie historique du latin, Paris.

GonzÁlez Rodríguez, Mํㅡㄹ 1986 Las unidades organizativas indígenas del área indoeuropea de Hispania, Vitoria-Gasteiz (Anejo n. ${ }^{\circ} 2$ de Veleia).

EADEM 2000/2002 «Die lateinische Epigraphie Hispaniens als Quelle für die keltische Gesellschaft und Religion» Veleia 18-19 39-60.

DE Hoz Bravo, Javier/Fernández Palacios, Fernando 2002 «Band-», Religiões da Lusitânia: loquuntur saxa, ed. J. Cardim Ribeiro, Lisboa. 45-52.

LEJEUnE, Michel 1982 «Notes d'étymologie gauloise» Études celtiques 191982 107$-111$.

MARCo Simón, Francisco 1998 Die Religion im keltischen Hispanien, Budapest.

IDEM 1999 «Divinidades indígenas en la Hispania indoeuropea» Veleia 16 33-49. MAYRHOFER, Manfred 1993 Etymologisches Wörterbuch des Altindoarischen vol. II/13, Heidelberg.

MorANDI, Alessandro 2000 «Sulla edizione dei testi epigrafici celtici d'Italia» Rivista di studi liguri anno 66 5-21.

Olivares Pedreño, Juan Carlos 2001 «Teónimos y pueblos indígenas hispanos: los Vettones» Iberia 4 57-69.

IDEM 2000-2001 «Teónimos y fronteras étnicas: los Lusitani» Lucentum 19-20 245-256. IDEM 2002 Los dioses de la Hispania céltica, Madrid y Alicante.

Pedrero SAncho, Rosa 1997/1999 «Aproximación lingüística al teónimo lusitanogallego Bandue/Bandi», Pueblos, lenguas y escrituras en la Hispania prerromana = Actas del VII Coloquio sobre lenguas y culturas paleohispánicas, edd. F. Villar/F. Beltrán, Salamanca. 535-543.

EADEM 1999/2001 «Los epítetos del teónimo occidental Bandue/i», Religión, lengua y cultura prerromanas de Hispania $=$ Actas del $8 .^{\circ}$ Coloquio sobre lenguas y culturas prerromanas de la Península Ibérica, edd. F. Villar/M P. Fernández Álvarez, Salamanca. 541-560.

PRÓSPER, Blanca Mª 2002 Lenguas y religiones prerromanas del occidente de la Península Ibérica, Salamanca. 
212 P. De Bernardo Stempel, Los formularios teonímicos, Bandus con Bandua

Redentor, Armando 2002 Epigrafia romana na região de Bragança, Trabalhos de Arqueologia 24.

SASTRE PRATS, Inés 2002 Onomástica y relaciones políticas en la epigrafía del Conventus Asturum durante el alto imperio, Anejos del AEspA 25.

SpicKermanN, W./WIEGELs, R. (eds.), Keltische Götter im römischen Reich, Akten des 4. internationalen F.E.R.C.AN Workshops in Osnabrück (4.-6. Oktober 2002), Osnabrücker Forschungen zu Altertum und Antike-Rezeption 9, Möhnesee 2004.

ZACCARIA, Claudio 2000/2002 «Alla ricerca di divinità 'celtiche' nell'Italia settentrionale in età romana. Revisione della documentazione per le regiones IX, X, XI» Veleia 18-19, 129-164. 